\title{
Effects of the secondary decays on the isotopic thermometers
}

\author{
Al. H. Raduta and Ad. R. Raduta \\ National Institute of Physics and Nuclear Engineering, \\ Bucharest, POB MG-6, Romania
}

\begin{abstract}
The sharp microcanonical multifragmentation model from [Al. H. Raduta and Ad. R. Raduta, Phys. Rev. C 55, 1344 (1997); Phys. Rev. C, in press] is employed for evaluating the nuclear caloric curve predictions of nine isotopic thermometers for three representative nuclei. Evaluations are performed for both primary decay and asymptotic stages. Effects of the secondary decays on the primary decay caloric curves are evidenced and discussed. In both cases a dispersive character of the isotopic caloric curves with increasing the source excitation energy is observed. A procedure of calibrating the isotopic thermometers on the microcanonical predictions for both primary decay and asymptotic stages is proposed. The resulting set of calibrating parameters for each thermometer is independent on the source size, on its excitation energy and, in the case of the primary decay, on the freeze-out radius assumption.
\end{abstract} PACS number(s): 25.70.Pq, 24.10.Pa 
Nuclear caloric curve is presently one of the most studied subjects in nuclear multifragmentation research. The reason lay in the resemblance of the nucleon-nucleon interaction with the van der Waals forces which motivates the expectation of a liquid-gas phase transition in nuclear matter. While statistical multifragmentation models (SMM [1] and MMMC [2]) predicted caloric curves with transition-like regions since 1985, the first experimental evaluation of the nuclear caloric curve was performed ten years later by the ALADIN collaboration [3]. A wide plateau interrupting the monotonical increase of the caloric curve at $5 \mathrm{MeV}$ temperature was evidenced with that occasion. This was interpreted as a sign of liquid-gas phase transition. Experiments of INDRA [4] and EOS [5] performed on other reactions followed showing sightly different results. Two years latter, papers of the ALADIN collaboration [6.7] announced a reevaluation of the neutron kinetic energies and of the energies of the spectator parts resulting in a modification of the ALADIN caloric curve from 1995. Reasons for such discrepancies lay in both the different employed reactions leading to different sequences of equilibrated sources $\left(E^{*}(A)\right)$ and, as shown above, in the degree of precision of the experimental measurements. The question is to what extent the experimentally measured caloric curves relate to the real one. Apart from the nonequilibrium parts of the excitation energies of the nuclear sources which have to be properly removed, one has to use a temperature formula which accounts for the finite size effects which manifest in nuclear systems.

In this respect, is widely used the Albergo isotopic formula [8], expressed as a double ratio of isotopic yields:

$$
T_{12 / 34}=\Delta B / \ln \left\{s\left[Y\left(A_{1}, Z_{1}\right) / Y\left(A_{2}, Z_{2}\right)\right] /\left[Y\left(A_{3}, Z_{3}\right) / Y\left(A_{4}, Z_{4}\right)\right]\right\},
$$

where $\Delta B=\left[B\left(A_{1}, Z_{1}\right)-B\left(A_{2}, Z_{2}\right)\right]-\left[B\left(A_{3}, Z_{3}\right)-B\left(A_{4}, Z_{4}\right)\right], B\left(A_{i}, Z_{i}\right)$ and $Y\left(A_{i}, Z_{i}\right)$ are respectively the binding energy and the yield corresponding to the isotope $\left(A_{i}, Z_{i}\right)$, $s$ is a statistical factor deduced from the ground state spin and masses of the isotopes and, finally, the isotope pairs $(1,2)$ and $(3,4)$ must differ by the same number of neutrons and/or protons. Being deduced on grandcanonical grounds, formula (1) does not account 
for finite size effects. Pochodzalla et al. [3] tried to remediate this drawback by including a calibrating factor $f_{T}=1.2$ chosen as to average the results of the QSM, GEMINI and MMMC models. Obviously, the efficiency of this method depends on the compatibility of the employed model with the physical phenomenon. Due to the finite dimensions of the systems currently involved, the microcanonical ensemble is the best solution. Moreover, one has to take into account the process of secondary decays from the primary decay excited fragments which modify the yields entering formula (11).

The calibration of seven isotopic thermometers on the prediction of the sharp microcanonical model from Ref. [9] was described in [10]. There, the Albergo thermometers were only microcanonically corrected. In order to allow a direct application of the thermometers on the experimental yields one has to apply a second correction for eliminating the effects of the secondary decays. This is now possible with the new version [11] of the sharp microcanonical multifragmentation model [9]. In comparison with its initial formulation, in Ref. [11] the microcanonical model is refined and improved by considering the experimental energy levels for fragments with $A \leq 6$ (instead of the level density Fermi gas formula) and by including the secondary decay stage. Using this version, a very good agreement was obtained in Ref. [1] between the predicted uncorrected HeLi caloric curve and the experimental (uncorrected) HeLi caloric curve of ALADIN from 1997 [6],

The present paper intends to use the new version [11] of the sharp microcanonical model [9] in order to analyze the effects of the secondary decays on the primary decay isotopic caloric curve and to perform a microcanonical calibration of nine isotopic thermometers for both primary decay and asymptotic stages. Also, a study is made concerning the sensitivity of the resulting parametrization to the freeze-out radius assumption.

Isotopic caloric curve evaluations are presented in Fig. 1 for nine isotopic thermometers: ${ }^{6,7} \mathrm{Li} /{ }^{3,4} \mathrm{He},{ }^{7,8} \mathrm{Li} /{ }^{3,4} \mathrm{He},{ }^{8,9} \mathrm{Li} /{ }^{3,4} \mathrm{He},{ }^{12,13} \mathrm{C} /{ }^{3,4} \mathrm{He},{ }^{13,14} \mathrm{C} /{ }^{3,4} \mathrm{He},{ }^{11,12} \mathrm{~B} /{ }^{3,4} \mathrm{He},{ }^{12,13} \mathrm{~B} /{ }^{3,4} \mathrm{He}$, ${ }^{1,2} \mathrm{H} /{ }^{3,4} \mathrm{He},{ }^{2,3} \mathrm{H} /{ }^{3,4} \mathrm{He}$ together with the microcanonical caloric curve, in the case of three representative source nuclei $(70,32),(130,54)$ and $(190,79)$ and a freeze-out radius parameter of $2.5 A^{1 / 3} \mathrm{fm}$. Calculations have been performed for both primary decay stage (left col- 
umn) and asymptotic stage (right column). In all cases the isotopic curves show a dispersive character growing with the increase of the source excitation energy. An interesting effect is the strong diminish of this dispersion after the secondary decays (see Fig. 1, right column). When analyzing the asymptotic curve this effect may induce the exaggerate impression that the uncorrected Albergo isotopic thermometers give quite close results and consequently, the finite size effects affecting formula (11) are small. In fact, secondary decays can bring some isotopic caloric curves quite close to the microcanonical ones in spite of the great deviation between the above mentioned curves appearing in the primary decay stage (e.g. the case of the ${ }^{13,14} \mathrm{C} /{ }^{3,4}$ He curve).

In order to calibrate formula (1) on the microcanonical predictions for both primary decay and asymptotic stages we apply the method described in Ref. [10]. First, the calibrating factors $f_{T}=T_{\text {micro }} / T_{\text {iso }}$ (where $T_{\text {micro }}$ denotes the microcanonical temperature and $T_{\text {iso }}$ the corresponding isotopic temperature) corresponding to each temperature point from Fig. 1 are represented in Fig. 2. In all cases, the $f_{T}\left(E_{e x}\right)$ curves are almost straight so one can use linear expressions for fitting them:

$$
f_{T}\left(E_{e x}\right)=a E_{e x}+b
$$

where $a$ and $b$ are calibrating parameters. A set of calibrating parameters $a, b$ was derived for each considered thermometer for both the primary decay and the asymptotic situations. The values of these parameters are given in Table 1 for the case of the primary decay and in Table [1] for the asymptotic case.

In order to check the efficiency of the obtained parametrization, the isotopic caloric curves corresponding to both primary decay and asymptotic situations (Fig. 1) are adjusted using the obtained calibration parameters according to the relation:

$$
T_{i s o}^{c o r r}\left(E_{e x}\right)=T_{i s o}\left(E_{e x}\right) f_{T}\left(E_{e x}\right) .
$$

The result is the overlapping between all the isotopic caloric curves and the microcanonical one for both the primary decay stage and the asymptotic stage presented in Fig. 3. In 
conclusion, $f_{T}\left(E_{e x}\right)$ is perfectly applicable for calibrating formula (11) correctly. It is remarkable that the obtained parameters do not depend on the source size or its excitation energy. This makes them quite applicable on experimental data where the source size is strongly dependent on its excitation energy. While the parametrization corresponding to the primary decay case (Table $\mathbb{\text { I }}$ ) needs a further correction as to erase the effects of the secondary decays, the parameterization corresponding to the asymptotic situation (Table III) is directly applicable on the experimental isotopic yields.

The modifications brought by the secondary decays to the primary decay isotopic caloric curve are clearly evidenced in Fig. 4. There it is represented the ratio between the primary decay caloric curves and the asymptotic caloric curve for all cases except ${ }^{13,14} \mathrm{C} /{ }^{3,4} \mathrm{He}$. For the clarity of the figure, this ratio can be very well approached by the ratio of the factors $f_{T}\left(E_{e x}\right)$ corresponding to the asymptotic situation and those corresponding the the primary decay situation, $f_{T}^{a s y m p}\left(E_{e x}\right) / f_{T}^{p r i m}\left(E_{e x}\right)$. Except for one situation $\left({ }^{6,7} \mathrm{Li} /{ }^{3,4} \mathrm{He}\right)$, the curves after the secondary decays are lower than those corresponding to the primary decay on the entire considered domain of excitation energy. Also, the deviation of the asymptotic curves from the primary decay curves increases in five cases $\left({ }^{7,8} \mathrm{Li} /{ }^{3,4} \mathrm{He},{ }^{8,9} \mathrm{Li} /{ }^{3,4} \mathrm{He},{ }^{12,13} \mathrm{C} /{ }^{3,4} \mathrm{He},{ }^{11,12} \mathrm{~B} /{ }^{3,4} \mathrm{He},{ }^{12,13} \mathrm{~B} /{ }^{3,4} \mathrm{He}\right)$ and decreases in other three cases $\left({ }^{6,7} \mathrm{C} /{ }^{3,4} \mathrm{He},{ }^{1,2} \mathrm{H} /{ }^{3,4} \mathrm{He},{ }^{2,3} \mathrm{H} /{ }^{3,4} \mathrm{He}\right)$.

Finally, a study is made concerning the influence of the freeze-out radius assumption on the resulting parametrization. To this aim, the isotopic caloric curves corresponding to the primary decay for the $(70,32)$ source for the freeze-out radii $2.25 A^{1 / 3} \mathrm{fm}$ and 2.75 $A^{1 / 3} \mathrm{fm}$ are plotted in the left column of Fig. 5. The overall aspect is maintained except the position of the plateau-like region. In the left column of Fig. 5 the caloric curves corresponding to each considered freeze-out radius, adjusted according to equation (3) using

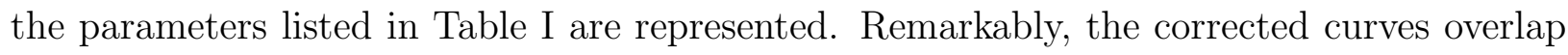
over the microcanonical ones. This means that the parametrization corresponding to the primary decay (Table $\mathbb{\square}$ ) is also independent to the freeze-out radius assumption. A similar study concerning the asymptotic isotopic caloric curves using the parameters from Table 
[11 showed the same tendency of overlapping between the isotopic curves but a small global deviation from the microcanonical curve was present for the $2.25 A^{1 / 3}$ case.

Summarizing, isotopic caloric curves have been evaluated for nine isotopic thermometers in both primary decay and asymptotic stages. In both cases a dispersive character of the isotopic caloric curve monotonically increasing with the increase of the excitation energy is evidenced. In the asymptotic stage this dispersion is strongly diminished. A set of microcanonical calibrating parameters was deduced for each of the considered thermometers for both primary decay and asymptotic situations. Remarkably, these parameters are independent on the dimension of the source, on its excitation energy and, in the primary decay stage, on the freeze-out radius assumption. Finally, a study was made concerning the modifications brought by the secondary decays to the primary decay isotopic caloric curves. 


\section{TABLES}

\begin{tabular}{lcr}
\hline \hline Thermometer & $\mathrm{a}\left(\right.$ nucleon $\left.\times \mathrm{MeV}^{-1}\right)$ & $\mathrm{b}$ \\
\hline${ }^{6,7} \mathrm{Li} /{ }^{3,4} \mathrm{He}$ & 0.016510 & 1.328440 \\
${ }^{7,8} \mathrm{Li} /{ }^{3,4} \mathrm{He}$ & 0.002526 & 1.004070 \\
${ }^{8,9} \mathrm{Li} /{ }^{3,4} \mathrm{He}$ & -0.011714 & 0.916471 \\
${ }^{12,13} \mathrm{C} /{ }^{3,4} \mathrm{He}$ & 0.016886 & 1.061580 \\
${ }^{11,12} \mathrm{~B} /{ }^{3,4} \mathrm{He}$ & -0.013010 & 0.880336 \\
${ }^{12,13} \mathrm{~B} /{ }^{3,4} \mathrm{He}$ & 0.000098 & 1.023200 \\
${ }^{1,2} \mathrm{H} /{ }^{3,4} \mathrm{He}$ & 0.011133 & 1.004980 \\
${ }^{2,3} \mathrm{H} / 3,4 \mathrm{He}$ & 0.013426 & 1.004870 \\
\hline \hline
\end{tabular}

TABLE I. Calibrating parameters corresponding to the primary decay stage.

\begin{tabular}{lcr}
\hline \hline Thermometer & $\mathrm{a}\left(\right.$ nucleon $\left.\times \mathrm{MeV}^{-1}\right)$ & $\mathrm{b}$ \\
\hline${ }^{6,7} \mathrm{Li} /{ }^{3,4} \mathrm{He}$ & -0.004475 & 1.492390 \\
${ }^{7,8} \mathrm{Li} /{ }^{3,4} \mathrm{He}$ & 0.043046 & 1.233270 \\
${ }^{8,9} \mathrm{Li} /{ }^{3,4} \mathrm{He}$ & -0.005079 & 1.081550 \\
${ }^{12,13} \mathrm{C} /{ }^{3,4} \mathrm{He}$ & 0.024806 & 1.333520 \\
${ }^{11,12} \mathrm{~B} /{ }^{3,4} \mathrm{He}$ & 0.016407 & 1.133040 \\
${ }^{12,13} \mathrm{~B} /{ }^{3,4} \mathrm{He}$ & 0.018067 & 1.254630 \\
${ }^{1,2} \mathrm{H} /{ }^{3,4} \mathrm{He}$ & -0.002311 & 1.318080 \\
${ }^{2,3} \mathrm{H} /{ }^{3,4} \mathrm{He}$ & 0.000675 & 1.235100 \\
\hline \hline
\end{tabular}

TABLE II. Calibrating parameters corresponding to the asymptotic stage. 


\section{FIGURES}
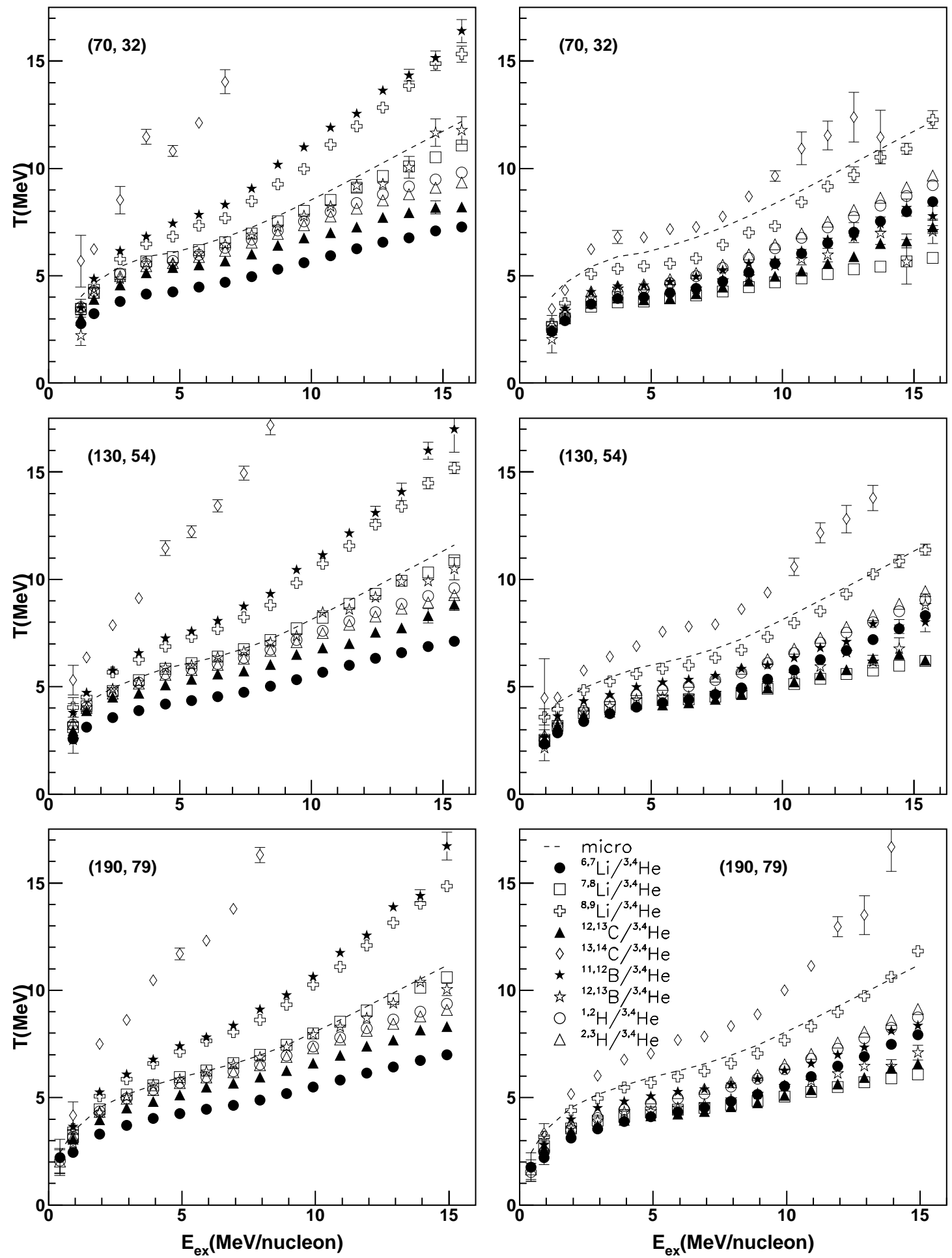

FIG. 1. Caloric curves corresponding to nine isotopic thermometers evaluated for three nuclear sources with the freeze-out radius $\mathrm{R}=2.5 \mathrm{~A}^{1 / 3} \mathrm{fm}$ in both primary decay (left column) and asymptotic (right column) stages. The microcanonical caloric curve is represented by a dashed line. 

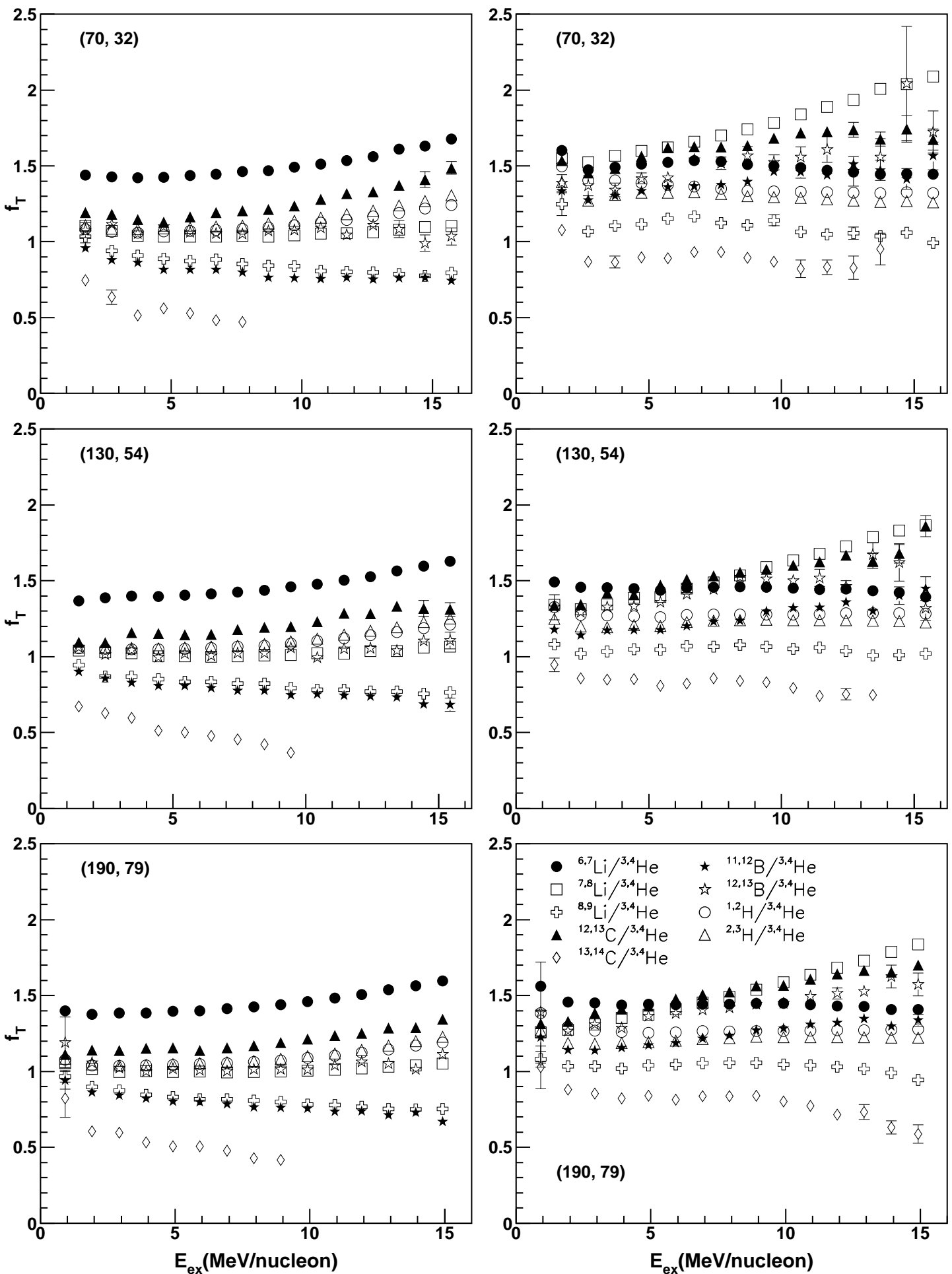

FIG. 2. Calibration factors corresponding to nine isotopic thermometers evaluated for three nuclear sources with the freeze-out radius $\mathrm{R}=2.5 \mathrm{~A}^{1 / 3} \mathrm{fm}$ in both primary decay (left column) and asymptotic (right column) stages. 

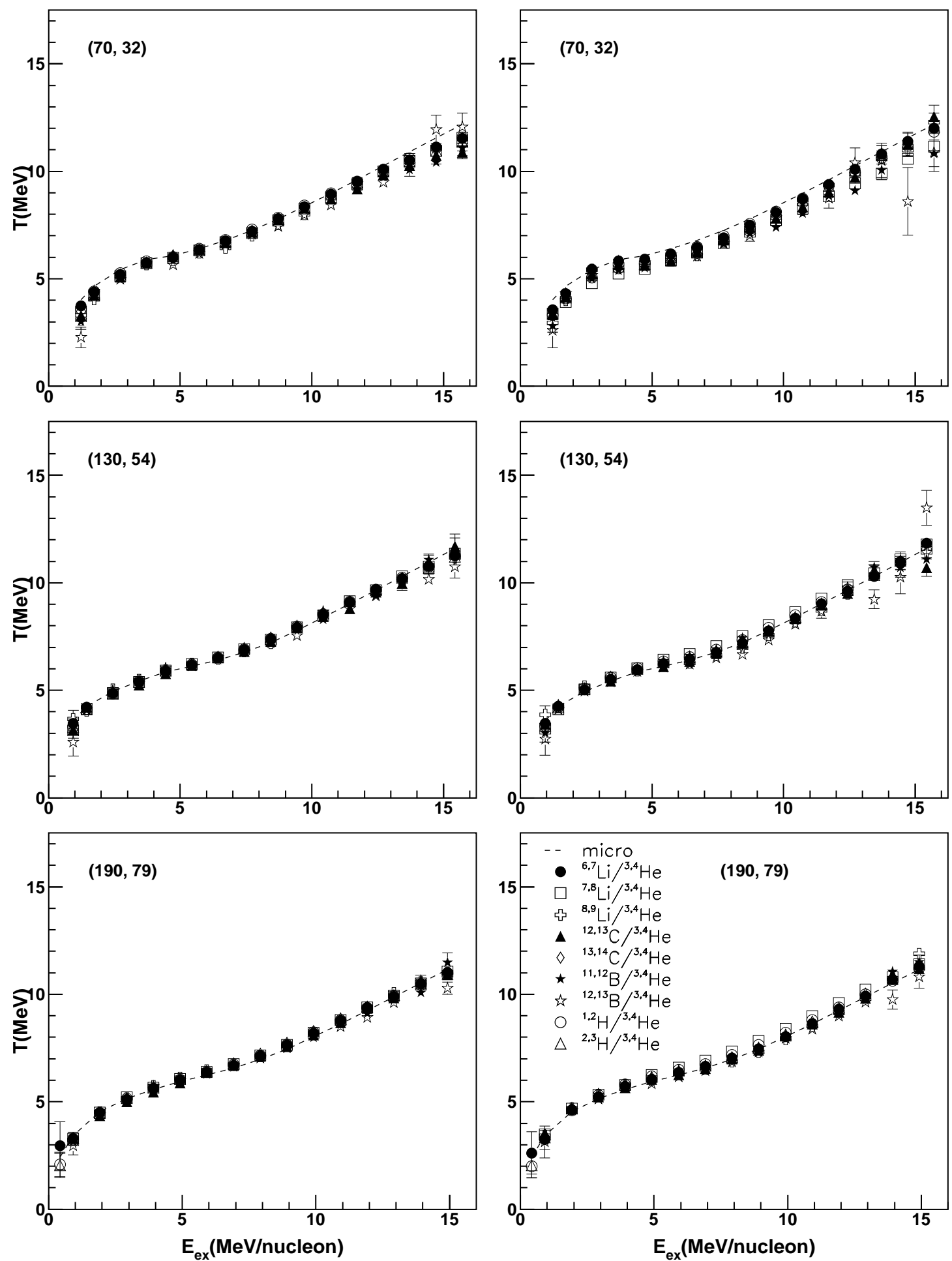

FIG. 3. The isotopic caloric curves from Fig. 11 calibrated by means of equation (3) and the parameters from Tables 目 and II. The microcanonical caloric curve is represented by a dashed line. 


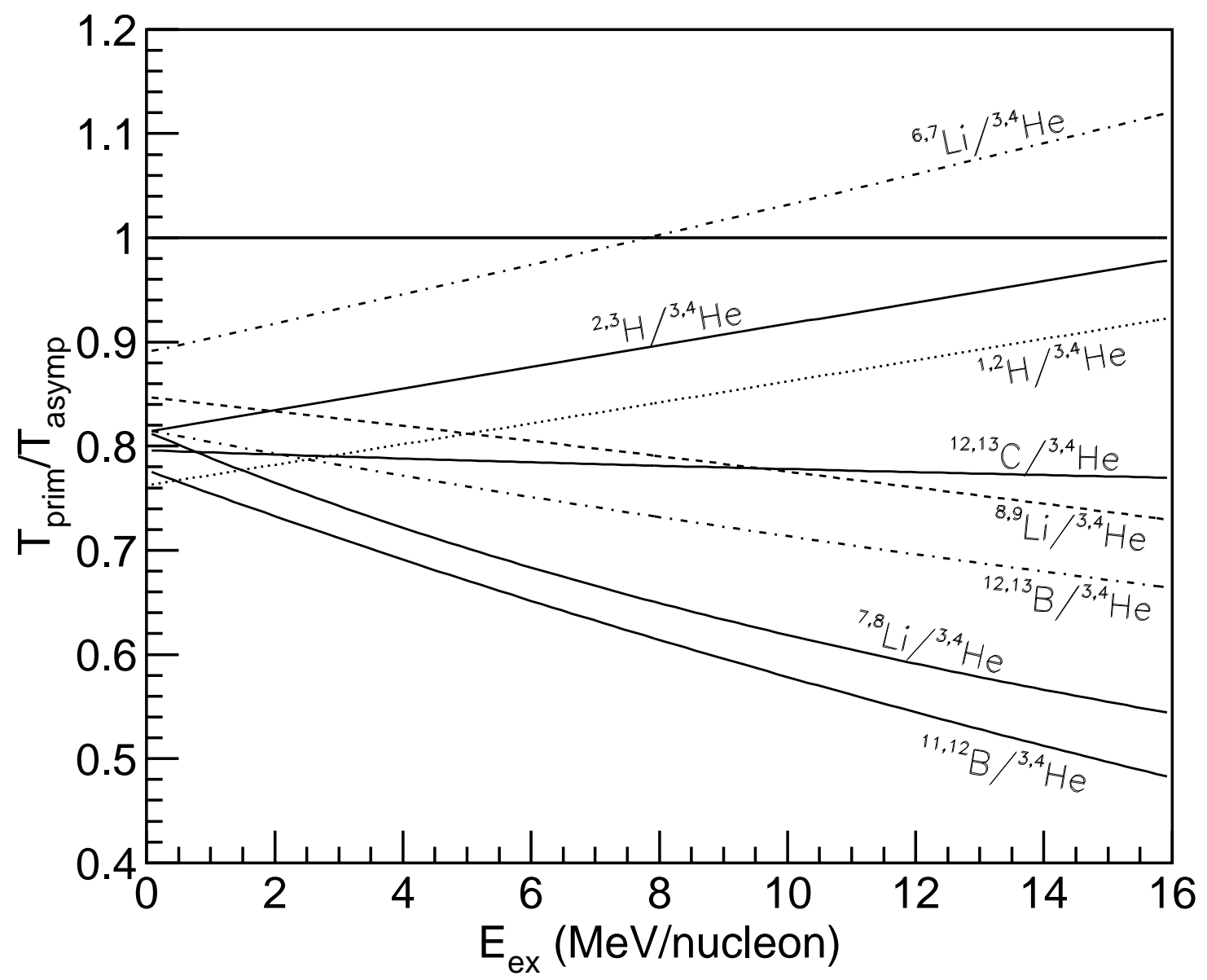

FIG. 4. Ratios between primary decay isotopic temperature and asymptotic isotopic temperature versus excitation energy approximated by the ratio $f_{T}^{a s y m p}\left(E_{e x}\right) / f_{T}^{\text {prim }}\left(E_{\text {ex }}\right)$ with $f_{T}\left(E_{e x}\right)$ given by equation (2) and the parameters $a$ and $b$ taken from Table [I. 

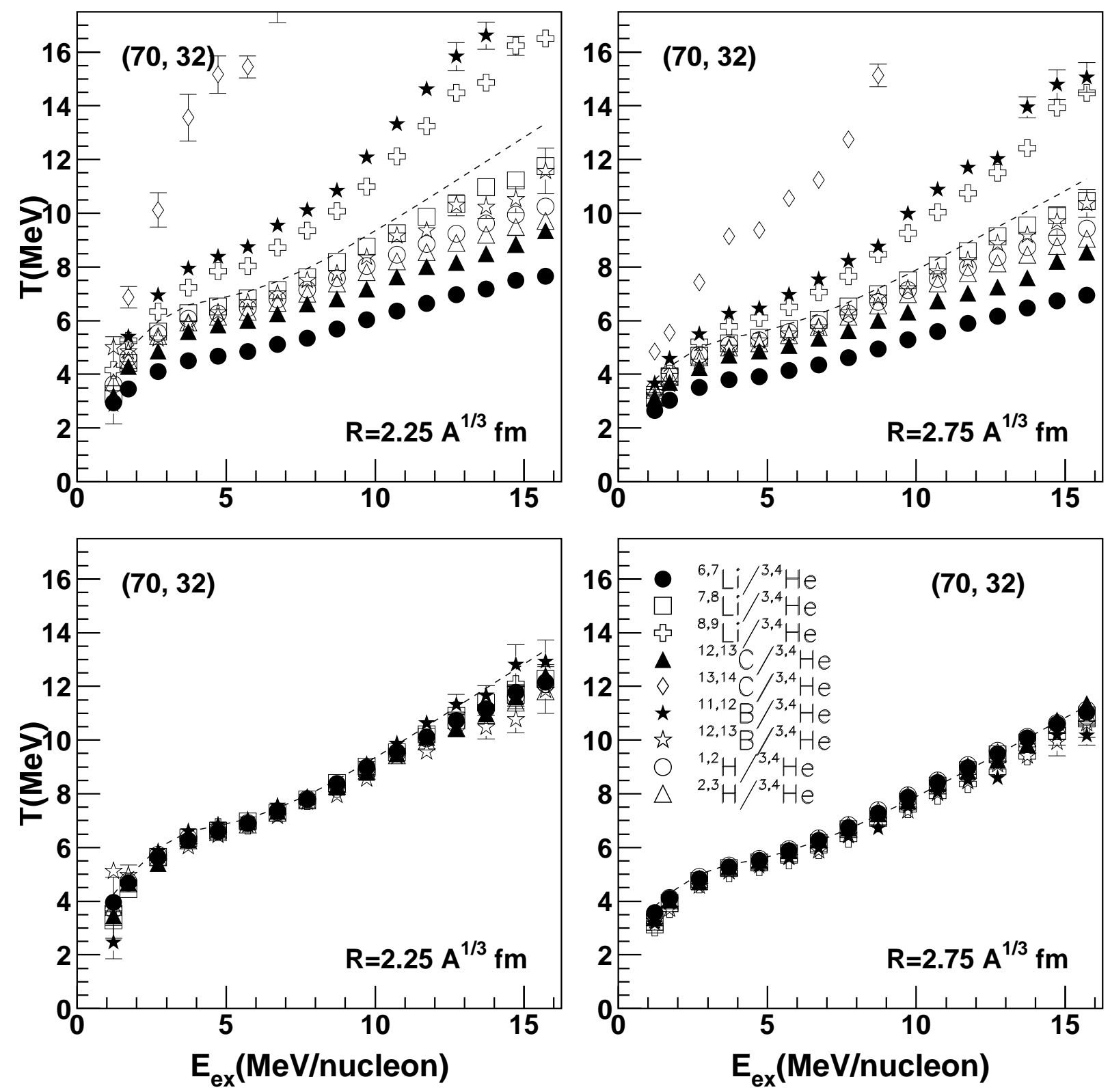

FIG. 5. Caloric curves corresponding to nine isotopic thermometers evaluated for the nuclear source $(70,32)$ and the freeze-out radii $2.25 \mathrm{~A}^{1 / 3} \mathrm{fm}$ and $2.75 \mathrm{~A}^{1 / 3} \mathrm{fm}$ (upper side) and the same

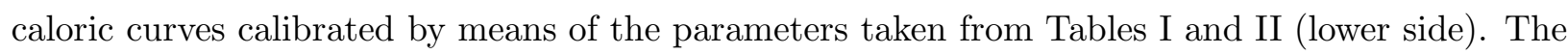
microcanonical caloric curve is represented by a dashed line. 


\section{REFERENCES}

[1] J. P. Bondorf, A. S. Botvina, A. S. Iljinov, I. N. Mishustin and K. Sneppen, Phys. Rep. 257, 133 (1995).

[2] D. H. E. Gross, Rep. Prog. Phys. 53, 605 (1990).

[3] J. Pochodzalla it et al., Phys. Rev. Lett. 75, 1040 (1995).

[4] Y. G. Ma et al., Phys. Lett. B 390, 41 (1997).

[5] J. A. Hauger et al., Phys. Rev. Lett. 77, 235 (1996).

[6] Hongfei Xi et al., Z. Phys. A 359, 397 (1997).

[7] W. Trautmann et al., GSI preprint 98-20, March 1998.

[8] S. Albergo, S. Costa, E. Costanzo and A. Rubbino, Il Nouvo Cimento 89A, 1 (1985).

[9] Al. H. Raduta and Ad. R. Raduta, Phys. Rev. C 55, 1344 (1997).

[10] Al. H. Raduta and Ad. R. Raduta, Phys. Rev. C 59, R1855 (1999).

[11] Al. H. Raduta and Ad. R. Raduta, Phys. Rev. C, in press. 